\title{
导模法生长微孔蓝宝石晶体工艺及性能研究
}

\author{
王东海 ${ }^{1}$, 薛艳艳 ${ }^{1}$, 李 纳 ${ }^{1}$, 周仕明 ${ }^{1}$, 徐晓东 ${ }^{2}$, 李东振 ${ }^{2}$, 徐 军 ${ }^{1}$, 王庆国 ${ }^{1}$
}

(1. 同济大学 物理科学与工程学院, 高等研究院, 上海 $200092 ; 2$. 江苏师范大学 物理与电子工程学院, 徐州 221116)

摘 要: 采用导模法生长了内孔径 $470 \mu \mathrm{m}$ 的蓝宝石晶棒和内孔径 $160 \mu \mathrm{m}$ 板形蓝宝石晶体。基于求解拉普拉斯方程 的数值解, 得到生长界面处微孔的熔体膜轮廓曲线, 采用插入钿丝的方法设计了生长模具以形成和维持晶体内的 微孔尺寸，同时解决了微孔蓝宝石长晶生长过程中的两个难点: (1)获得高质量的蓝宝石晶体; (2)在蓝宝石晶体中形 成并维持所需的内孔尺寸。所生长的晶体透明完整、无开裂、双晶摇摆曲线测定显示其衍射半峰宽为 $3.8^{\prime}$, 具有良 好的结晶质量

关 键 词: 微孔蓝宝石晶体; 导模法; 晶体生长

中图分类号: O782 文献标识码: A

\section{Micro-tube Sapphire Crystal Grown by the Edge-defined-film Fed Method}

\author{
WANG Dong-Hai ${ }^{1}$, XUE Yan-Yan ${ }^{1}$, LI Na $^{1}$, ZHOU Shi-Ming ${ }^{1}$, XU Xiao-Dong ${ }^{2}$, \\ LI Dong-Zhen ${ }^{2}$, XU Jun ${ }^{1}$, WANG Qing-Guo ${ }^{1}$
}

(1. Institute for Advanced Study, School of Physics Science and Engineering, Tongji University, Shanghai 200092, China; 2. School of Physics and Electronic Engineering, Jiangsu Normal University, Xuzhou 221116, China)

\begin{abstract}
Sapphire rod with micro-tube (inner diameter: $470 \mu \mathrm{m}$ ) and sapphire plate with micro-tube (inner diameter: $160 \mu \mathrm{m}$ ) were grown by the edge-defined-film fed method (EFG). Profile curves of the small liquid menisci were investigated on the base of numerical solution of the Young-Laplace equation. By inserting Mo wires, new type of die was designed to form and sustain size of micro-tube in the volume of the crystal. Two problems were solved at the same time: 1) obtaining high quality sapphire crystal;2) forming and sustaining necessary micro-tube sizes in the volume of the crystal. The crystal were transparent, colorless, no cracking and good XRC value of 3.8 arcmin, demonstrating the high quality of the as-grown crystal.
\end{abstract}

Key words: micro-tube sapphire crystal; edge-defined-film fed growth method; crystal growth

蓝宝石晶体由于具有优异的机械性能、电学性 能、光学性能和化学稳定性而被广泛应用于精密仪 表机械轴承、绝缘材料、窗口材料和光学元件材料 等方面。内部具有微孔(内径小于 $1 \mathrm{~mm}$ )的蓝宝石由 于其特殊的结构, 可用作高压核磁共振波谱的高灵

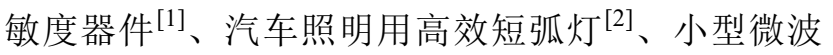

气体放电等离子体源，以及用来测量高温高压下的 液态金属、合金或石墨的热物理性质 ${ }^{[3]}$; 微孔蓝宝 石晶体同样被应用于肿瘤学, 在光动力学疗法和激 光热物理疗法中用微孔蓝宝石晶体作为光波导介质 将激光传输到肿瘤表面; 微孔蓝宝石晶体也可用作 智能手术刀 ${ }^{[4]}$, 同时具有切除和苂光诊断的功能。 
俄罗斯学者 ${ }^{[5]}$ 提议并尝试使用微孔蓝宝石晶体 作为太赫兹光子晶体波导, 为太赫兹波的传输问题 提供了一种有效的解决方案。台湾学者 Huang 等 ${ }^{[6]}$ 采用共拉激光加热基座法(Co-drawing laser-heated pedestal growth system)生长 $\mathrm{Cr}^{4+}$ : $\mathrm{YAG}$ 双包层光纤时 使用微孔蓝宝石晶体辅助加热, 成功地抑制了 $\mathrm{CO}_{2}$ 激光器加热功率的波动, 将纤芯的直径变化率降低 到未使用微孔蓝宝石的 $1 / 3$, 并将熔区的温度梯度 降低到原来的 $1 / 2.5$, 低温度梯度下生长的光纤纤芯 更光滑, 最终将光纤的传输损耗由 $0.6 \mathrm{~dB} / \mathrm{cm}$ 降低 到 $0.02 \mathrm{~dB} / \mathrm{cm}$ 。

由于蓝宝石硬度高, 难以加工, 特别是内孔径 小于 $1 \mathrm{~mm}$ 的微孔蓝宝石晶体, 用机械加工或激光 打孔的方法难以加工长度 $1 \mathrm{~cm}$ 以上的微孔。对于蓝 宝石这种难以加工的晶体, 最好是直接在晶体生长 过程中完成晶体的成型, 即通过晶体生长的方式获 得接近最终产品尺寸的晶体, 这恰好是导模法长晶 的特点。

导模法是利用与熔体浸润的模具将坩埚内的熔 体通过模具内毛细孔或毛细缝上升到模具上表面, 通过籽晶牵引在模具上表面进行晶体生长的技术, 生长出晶体的横截面形状取决于模具上表面的形 状。导模法已经成功地生长了片状(含多片)、棒状、 管状和纤维等形状的晶体。如今, 导模法已被大规 模用来产业化生产蓝宝石晶体、硅晶体和锗晶体等。 比较知名的导模法量产蓝宝石的企业或研究所有: 法国的圣戈班(Saint-Gobain)晶体公司、日本的京瓷 (Kyocera)和并木(NAMIKI)、俄罗斯的 EZAN RAS 公司等。

蓝宝石管是导模法生长的常规蓝宝石产品, 其 生长示意图如图 1 所示, 蓝宝石管内孔是由模具中 间的孔洞形成, 生长蓝宝石管时需避免熔体膜中的熔 体进入模具表面的孔洞中, 以形成中空的管状结构。

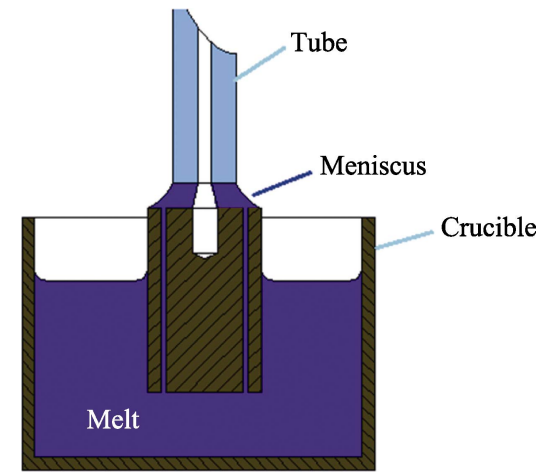

图 1 蓝宝石管生长示意图

Fig. 1 Schematic diagram of tube forming by a hollow in the die surface
目前已报道的导模法生长的最大尺寸的蓝宝石 圆管外径 $85 \mathrm{~mm}^{[7]}$, 最小尺寸的蓝宝石管内径为 $0.5 \mathrm{~mm}^{[4]}$, 生长微孔蓝宝石晶体的难点在于生长过 程中熔体很容易进入已生长的微孔中而将微孔堵塞, 这是因为微孔的直径远小于蓝宝石的毛细常数 $(6 \mathrm{~mm})$, 根据熔体沿毛细孔上升的高度公式(1):

$$
H=\frac{2 \gamma \cos \theta}{\rho r g}
$$

其中: $\gamma$ 为熔体表面张力系数 $(\mathrm{N} / \mathrm{cm}), \rho$ 为熔体密度 $\left(\mathrm{g} / \mathrm{cm}^{3}\right), r$ 为毛细管半径 $(\mathrm{cm}), g$ 为重力加速度 $(\mathrm{N} / \mathrm{g})$, $\theta$ 为固液润湿角。可见毛细孔越小, 孔内液面上升越 高, 当 $r=0.3 \mathrm{~mm}$ 时 $H=120 \mathrm{~mm}$, 已经生长的微孔极 易在生长过程中被模具表面熔体膜的熔体堵塞, 为 确保微孔的连续性，提拉速率只能在很小范围内变 化以维持较薄的熔体膜, 熔体膜厚度仅几十微米 (熔体膜厚度的减少即导致熔体对流的减少，使熔 体不致于进入模具表面的微小的孔洞，而丧失模具 长管的功能), 这种生长状态接近于过冷态, 这时晶 体极易与模具表面凝固在一起而导致与模具粘连, 只能通过升高模具温度使晶体与模具分离, 而升温 会增加熔体膜厚度, 使得熔体进入已生长的微孔中, 将微孔堵塞; 所以，国际知名的导模法生长蓝宝石 晶体的机构也难以生长内径小于 $0.5 \mathrm{~mm}$ 的蓝宝石 管, 见表 1 , 表中数据来源于各机构微孔蓝宝石最 小内径。

当生长具有较大横截面积的微孔蓝宝石晶体时, 形成和维持微孔的稳定生长面临更大的困难, 需同 时解决两个问题: 1)获得高质量的晶体; 2)维持微孔 尺寸的稳定生长。生长高质量蓝宝石晶体的前提是 在熔体膜稳定存在时，在结晶前沿维持尽可能高的 过热度 ${ }^{[8]}$ 。然而, 为形成维持熔体膜内部和固液界面 微孔的尺寸稳定性, 需要固液界面处于过冷态, 此 时, 熔体膜高度极小, 导致固液界面失稳, 以致晶 体缺陷产生并导致晶体横截面尺寸波动。

本工作通过自行设计的微孔晶体生长模具, 成 功生长出内孔径 $<0.5 \mathrm{~mm}$ 的微孔蓝宝石晶体, 最大 长度达 $220 \mathrm{~mm}$, 并对生长的微孔蓝宝石晶体进行 了性能测试。

表 1 各机构导模法生长微孔蓝宝石最小管内径参数

Table 1 Minimal inner sizes of sapphire tube grown by different companies with EFG method

\begin{tabular}{ccc}
\hline Company & Minimal tube ID size $/ \mathrm{mm}$ & Nation \\
\hline Tydex & 0.80 & Russia \\
Rostox-N & 0.50 & Russia \\
Namiki & 2.00 & Japan \\
Kyocera & 1.30 & Japan \\
Saint-gobain & 0.58 & France \\
Crytur & 0.60 & Australia \\
\hline
\end{tabular}




\section{1 实验方法}

\section{1 微孔蓝宝石晶体生长}

微孔蓝宝石晶体生长装置如图 2 所示, 模具毛 细孔内插入直径 $0.3 \sim 0.4 \mathrm{~mm}$ 的细钼丝, 钼丝高出模 具顶面约 $0.6 \sim 1 \mathrm{~mm}$; 坩埚内装入纯度 $99.99 \%$ 的氧 化铝原料, 生长气氛为流动氩气气氛, 生长方向为 $c$ 向, 生长速率为 $60 \sim 120 \mathrm{~mm} / \mathrm{h}$ 。

\section{2 性能表征}

采用 OLYMPUS 公司生产的 BX51 显微镜测量 了微孔的尺寸; 采用 QuanTA-200F 型环境扫描电 子显微镜(SEM)分析微孔蓝宝石径向截面微孔的形 貌和结构; 采用德国 Bruker 公司生产的 D8 DISCOVER 高分辨率 X 射线衍射仪测试了单晶样 品的摇摆曲线, 测试条件: $\mathrm{Cu} \mathrm{K \alpha}$ 辐射、工作电压 $40 \mathrm{kV}$ 、工作电流 $40 \mathrm{~mA}$ 、扫描步长 $0.02^{\circ}$ 。

\section{2 结果与讨论}

\section{1 晶体生长分析}

为研究和优化微孔蓝宝石晶体的生长过程, 对 微孔内部的熔体膜进行研究, 基于拉普拉斯方程, 对于圆环形的熔体膜具有如下无量纲形式 ${ }^{[9]}(2)$ :

$$
Z^{\prime \prime} r+Z^{\prime}\left(1+Z^{\prime 2}\right) \pm 2(d-Z)\left(1+Z^{\prime 2}\right)^{3 / 2} r=0
$$

其中: $Z(r)$ 为熔体膜轮廓曲线, $r$ 为自晶体对称中心向外 延伸的坐标, $d$ 为坩埚内自由液面和熔体膜底部的高度 差, 见图 3。计算时 $d=-4$ (与蓝宝石毛细常数归一化, 蓝宝石毛细常数 $a$ 为 $6 \mathrm{~mm})$ 当生长小直径晶体时 $(r<a=$ $6 \mathrm{~mm}$ ), 毛细作用远大于重力作用, 式(2)可以简化为(3):

$$
Z^{\prime \prime} r+Z^{\prime}\left(1+Z^{\prime 2}\right) \pm 2 d\left(1+Z^{\prime 2}\right)^{3 / 2} r=0
$$

式(3)的边界条件为(4):

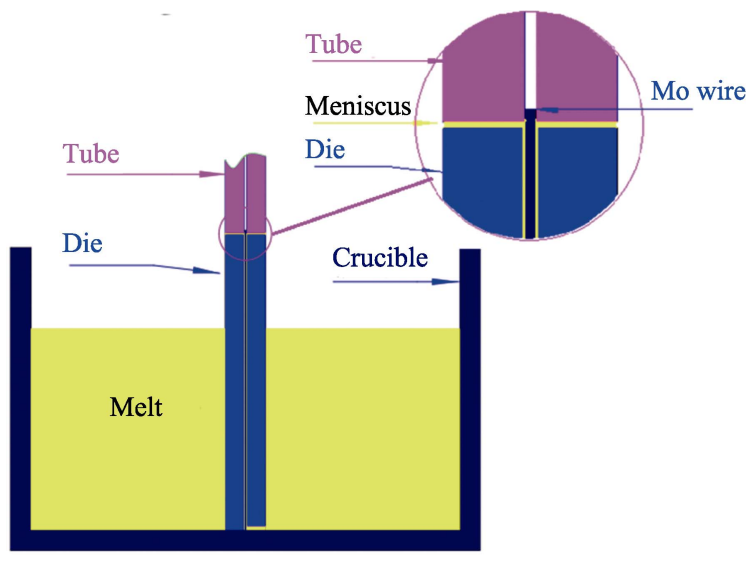

图 2 蓝宝石微孔生长示意图

Fig. 2 Schematic diagram of micro-tube forming

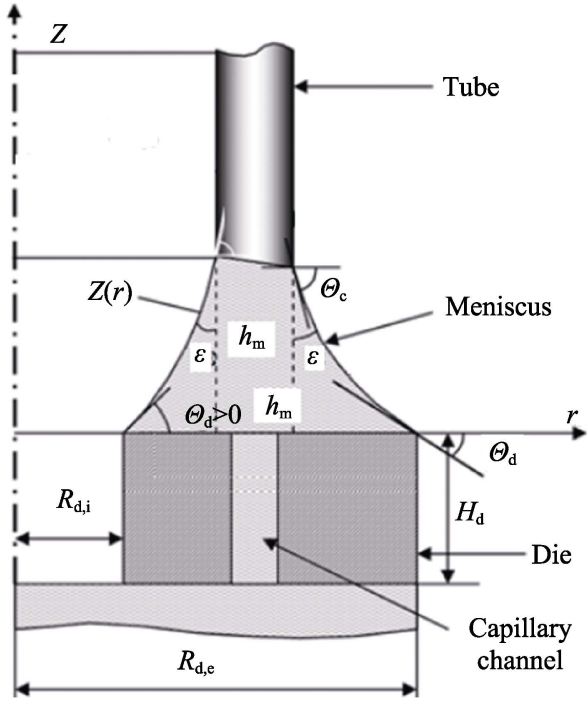

图 3 蓝宝石管生长计算模型

Fig. 3 Calculation model of sapphire tube growth

$$
Z\left(r_{\mathrm{d}}\right)=0,-\operatorname{arctg} Z^{\prime}\left(r_{\mathrm{c}}\right)=\pi / 2-\varepsilon
$$

其中: $r_{\mathrm{c}}$ 为晶体半径, $r_{\mathrm{d}}$ 为模具内孔半径, $\varepsilon$ 为材料生 长角 ([0001]方向蓝宝石晶体生长角大约为 $17^{\circ}$, [1010]方向蓝宝石生长角大约为 $35^{\circ[10]}$ 。因此, 模具 上表面处熔体膜高度为 0 , 熔体膜与蓝宝石晶体接 触位置的上边界满足生长角为常数的条件。

因为常规管状蓝宝石晶体报道的最小内径为 0.5 0.6 mm, 通过式(3)计算了内孔直径为 0.5856 $0.6 \mathrm{~mm}$ 的圆管内部的熔体膜轮廓曲线 $Z(r)$, 步长 为 $0.0012 \mathrm{~mm}$, 模具中心孔直径 (模具工作边)为 $0.582 \mathrm{~mm}$, 如图 4 所示, 从图中可以看出, 当内孔 直径为 $0.6 \mathrm{~mm}$ 时, 熔体膜厚度为 $0.048 \mathrm{~mm}$, 内孔直 径越小熔体膜厚度越小, 此时晶体生长过程处于过 冷态，晶体处于即将粘模具的临界状态。由于难以 控制整个模具表面温度均匀性，对于横截面积较大 的晶体很难控制。

通过计算得到生长外直径 $5.98 \mathrm{~mm}$, 内孔直径 $0.47 \mathrm{~mm}$ 的蓝宝石管熔体膜轮廓曲线, 如图 5 所示,

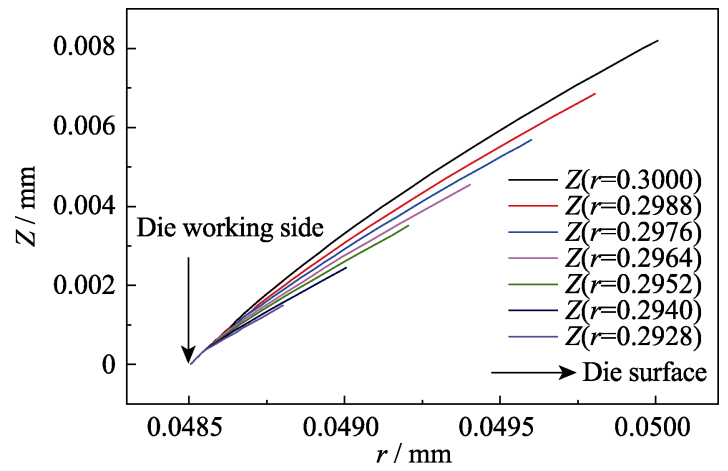

图 4 圆管内部的熔体膜轮廓曲线

Fig. 4 Profile curves of small internal menisci at different crystal radii 
从图中可以看出生长外直径 $5.98 \mathrm{~mm}$ 蓝宝石管时熔 体膜的厚度约 $0.820 \mathrm{~mm}$, 常规管状生长方法不再满 足内孔稳定形成的条件。由图 5 可见, 通过在模具 毛细孔中心插入钼丝, 并且钼丝露出模具的高度超 出熔体膜的厚度, 熔体膜的熔体只能在钼丝周围结 晶, 于是在钼丝的周围形成了中空的管状结构(微 孔), 晶体管与钼丝完全贴合, 杜绝了熔体进入已生 长的微孔的可能。

钼丝高于熔体膜的最优值为 $0.1 \sim 0.2 \mathrm{~mm}$, 过高 容易把钼丝从模具中拉出或拉断, 或者晶体由于受 到钼丝牵拉而弯曲变形, 晶体内部的微孔也随之弯 曲变形。钼丝露出模具高度低于熔体膜则不能形成 微孔, 只能形成晶棒, 如图 6 所示。

\section{2 晶体生长和显微镜分析}

图 7 为内孔径为 $0.47 \mathrm{~mm}$ 的微孔蓝宝石晶体照 片, 外部尺寸为 $\phi 5.98 \mathrm{~mm} \times 220 \mathrm{~mm}$; 晶体外形均 匀完整, 无开裂和多晶镶嵌结构, 无肉眼可见的宏 观气泡。显微镜下测量的中心孔直径为 $0.47 \mathrm{~mm}$, 晶 体横截面的 SEM 观察结果显示, 中心孔略呈椭圆 形，这是由于钼丝尖端形状不是规则圆形所致。

图 8 为内孔径为 $0.16 \mathrm{~mm}$ 的多微孔蓝宝石晶体 照片, 外形为平板结构, 内部具有 6 条微孔, 晶体外 形同样均匀完整、无开裂和多晶镶嵌结构, 显微镜 下测量的中心孔直径为 $0.16 \mathrm{~mm}$ 。

图 9 为内孔径为 $0.47 \mathrm{~mm}$ 的微孔蓝宝石晶体的

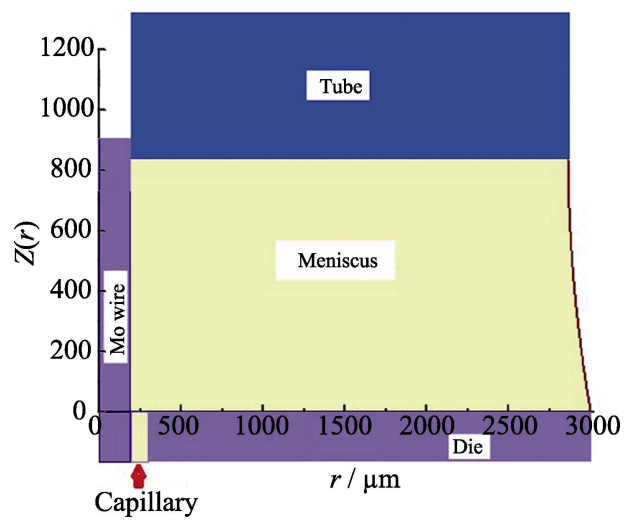

图 5 生长外直径 $5.98 \mathrm{~mm}$ 、内孔径 $0.47 \mathrm{~mm}$ 蓝宝石管熔体 膜轮廓曲线

Fig. 5 Profile curve of sapphire tube with outer diameter of $5.98 \mathrm{~mm}$ and inner diameter of $0.47 \mathrm{~mm}$

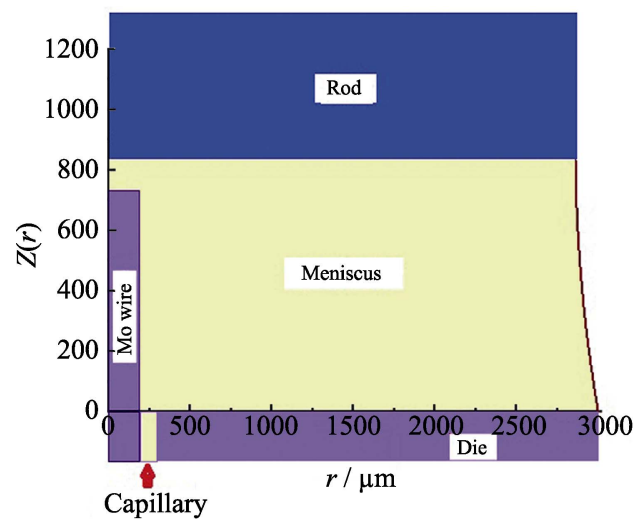

图 6 熔体膜高度高于钼丝, 生长出蓝宝石晶体棒

Fig. 6 A raise meniscus level and rod formation
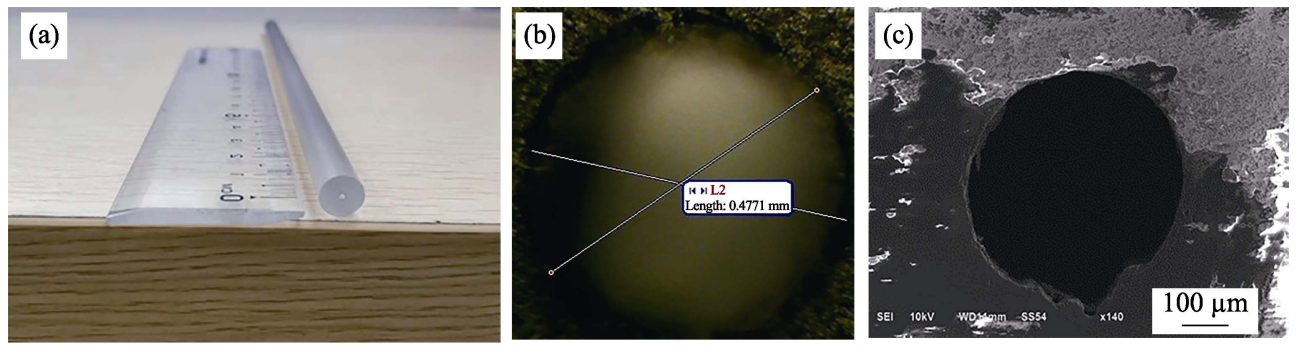

图 7 内孔径为 $0.47 \mathrm{~mm}$ 的微孔蓝宝石晶体照片(a)、显微镜测量结果(b)及 SEM 观察照片(c)

Fig. 7 (a) Photo of as-grown micro-tube sapphire with the inner diameter of $0.47 \mathrm{~mm}$; (b) Microscopic photo of the as-grown micro-tube sapphire; (c) SEM image of the as-grown micro-tube sapphire
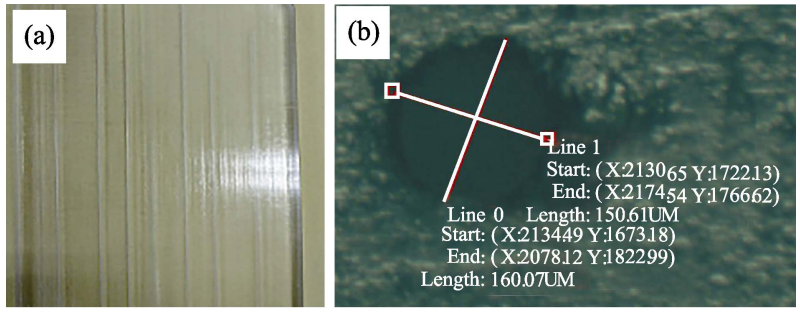

图 8 (a) 内孔径为 $0.16 \mathrm{~mm}$ 的微孔蓝宝石晶体照片; (b)显 微镜测量内孔径测试结果

Fig. 8 (a) Photo of as-grown micro-tube sapphire with the inner diameter of $0.16 \mathrm{~mm}$; (b) Microscopic photo of the as-grown micro-tube sapphire

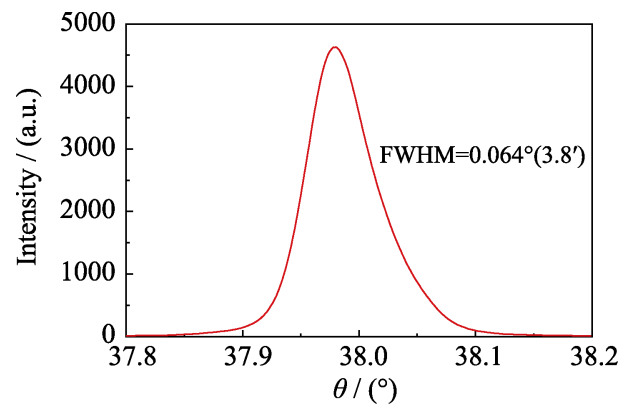

图 9 生长的微孔蓝宝石晶体的 $\mathrm{X}$ 射线双晶摇摆曲线

Fig. 9 X-ray rocking curve of the as-grown micro-tube sapphire crystal 
双晶摇摆曲线, 半峰宽仅 3.8', 摇摆曲线峰形对称 性好, 表示晶体结晶质量较高、单晶性好。

\section{3 结论}

本工作基于求解拉普拉斯方程的数值解, 研究 了蓝宝石微孔内径和熔体膜厚度的关系, 从而优化 生长工艺。通过在模具毛细孔中插入钼丝这一方式, 成功解决了微孔蓝宝石长晶生长过程中的两个难点: (1)获得高质量的蓝宝石, (2)在蓝宝石棒或晶片中形 成并维持所需的内孔尺寸; 最终生长出了内径 0.47 和 $0.16 \mathrm{~mm}$ 微孔蓝宝石晶体, 晶体透明完整、无开 裂、双晶摇摆曲线测定显示其衍射半峰宽为 $3.8^{\prime}$, 具 有良好的结晶完整性, 这有助于拓展微孔蓝宝石晶 体在太赫兹波传输等领域的应用。

\section{参考文献:}

[1] AMOLD M R, KALBITZER H R, KREMER W. High-sensitivity sapphire cells for high pressure NMR spectroscopy on proteins. $J$.
Magn. Reson., 2003, 161(2): 127-131.

[2] KetTlitZ M, WENDT M, SCHNEIDENBACH H, et al. Plasma diagnostics in $\mathrm{Hg}$-free short-arc lamps for automotive lighting. $J$. Phys. D Appl. Phys., 2007, 40(13): 3829-3835.

[3] SAVVATIMSKI A I, FORTOV V E, CHERET R. Thermophysical properties of liquid metals and graphite, and diamond production under fast heating. High Temp-High Press, 1998, 30(1): 1-18.

[4] KURLOV V N, SHIKUNOVA I A, RYABOVA A V, et al. Sapphire smart scalpel. Aip. Conf. Proc., 2010, 1226: 76-81.

[5] ZAYTSEV K I, KATYBA G M, KURLOV V N, et al. Terahertz photonic crystal waveguides based on sapphire shaped crystals. IEEE Tran. Thz. Sci. Techn., 2016, 6(4): 576-582.

[6] HUANG K Y, HSU K Y, JHENG D Y, et al. Low-loss propagation in $\mathrm{Cr}^{4+}$ : YAG double-clad crystal fiber fabricated by sapphire tube assisted CDLHPG technique. Optics Express, 2008, 16(16): 12264-12271.

[7] KURLOV V N, EPELBAUM B M. EFG growth of sapphire tubes up to $85 \mathrm{~mm}$ in diameter. J. Crystal Growth, 1998, 187(1): 107-110.

[8] PEROV V F, PAPKOV V S, IVANOV I A. Defects in sapphire tapes obtained by Stepanov method. Izv. Akad. Nauk SSSR, Ser. Fiz., 1979, 43(9): 1977-1981.

[9] DHANARAJ G, BYRAPPA K, PRASAD V, et al. Springer Handbook of Crystal Growth. 2010: 514-515.

[10] DREEBEN A B, KIM K M, SCHUJKO A, Measurement of meniscus angle in laser heated float zone growth of constant diameter sapphire crystals. J. Crystal Growth, 1980, 50: 126-132. 